\title{
Research Paper \\ Design, Implementation, and Assessment of Virtual Training of Dental Students in Elderly Dentistry Topic
}

\author{
Katayoun Sargeran ${ }^{1} \oplus,{ }^{*}$ Samaneh Razeghi ${ }^{1}$, Zohreh Khorshidi ${ }^{1}$
}

1. Department of Community Oral Health, School of Dentistry, Tehran University of Medical Sciences, Tehran, Iran.

Received: 16 Oct. 2017 Accepted: 01 Feb. 2018

Key words:

Geriatric dentistry, Traditional training, Virtual training dtation: Sargeran K, Razeghi S, Khorshidi Z. [Design, Implementation, and Assessment of Virtual Training of Dental Students in Elderly Dentistry Topic (Persian)]. Iranian Journal of Ageing. 2018; 13(1):4-15. https://doi.org/10.21859/SIJA.13.1.4

dot: : https://doi.org/10.21859/SIJA.13.1.4

\begin{abstract}
Objectives To achieve better oral health care for the growing elderly population it requires education of dentists who have attitudes; knowledge and skills. With regard to the benefits of web-based training, the present study aimed to design, implement, and evaluate a short virtual course in geriatric dentistry for the senior dental students of Tehran University of Medical Sciences and compare it with traditional method.

Methods \& Materials In the present interventional study, 64 senior dental students participated. In one group $(n=31)$ educational content presented through virtual method and in the other group $(n=33)$ via traditional manner. A standard questionnaire was completed by both groups before and after the educational intervention. The questionnaire included questions on general knowledge and attitudes of aging and knowledge, attitude and performance about geriatric oral health. Statistical analysis was conducted with SPSS software using covariance and regression tests.

Results No significant difference found between the mean scores of general knowledge and attitudes of aging and knowledge and attitudes about geriatric oral health of two groups. The mean scores of geriatric oral health practice in the traditional and virtual groups increased from $45.4+20$ to $56.9+26$ and from $52.9+20$ to $75.4+21$, respectively, after the intervention $(P<0.05)$. Regression analysis on the scores of students before the intervention showed that the general attitude of aging was related to "living with the elderly at the present time," however no significant relationship was observed in other areas such as between students' scores and their background factors.

Conclusion The virtual method is effective in gaining educational insights about geriatric dentistry compared with traditional method and can be used as an alternative for teaching gerodontology to dental students.
\end{abstract}

\section{Extended Abstract}

\section{Objectives}

iven the increasing number of elderly people, it is essential for dentists to receive necessary training to prevent and treat oral diseases in this group of people. The topic of gerontology has just become a theoretical unit which has been added to the dental curriculum in Dentistry Colleges in Iran and in that direction those colleges are planning educational programs. On the other hand, with the development of information technology, medical education methods have been transformed and currently use of newer methods such as virtual tutorials is being stressed for

\section{* Corresponding Author:}

Samaneh Razeghi, MD

Address: Department of Community Oral Health, School of Dentistry, Tehran University of Medical Sciences, Tehran, Iran.

Tel: +98 (912) 3542871

E-mail: s-razeghi@tums.ac.ir 
knowledge transfer [1]. Reducing spatial and temporal constraints for education, validating repeatability of the learning process, tracking student activity more easily, and changing teacher-centered to student-centered education are the benefits of virtual education [2]. Therefore, this study was conducted with the aim of design, implementation, and evaluation of virtual education of elderly dentistry topic for dentistry graduate students of Medical University of Tehran in the academic year of 2016-2017 and comparing it with the traditional method.

\section{Methods and Materials}

The Ethics Committee of Tehran University of Medical Sciences confirmed this study with no. IR.TUMS. REC.1395.2415. In this study, graduate students in Tehran University of Dentistry was evaluated by census method. A total of $64(82 \%)$ out of 78 students participated in the study. Traditional training group and virtual education group comprised of 33 and 31 students, respectively. Training program was performed as a workshop in three sessions and introduced within a week in the form of the society-centered credit 3. Data were collected using pre-test/post-test design (pre-test, before the first session in both groups; post-test, after the third session in both groups).

At the first session of educational content, two 45-minute speeches were presented by two professors from the Department of Oral Health and Social Dentistry for traditional teaching group. In the virtual training group, the content placed on the system of university symbols. The educational content provided was same in both groups. Second session of the educational program was carried out for students in both traditional and virtual groups in the form of an educational field (urban health center affiliated to Tehran municipality). Students of both groups (separately) tried filed examination, correct communication, receiving biographies, oral health assessment, studying the therapeutic needs, and providing elderly health education. At the third session, students of both groups provided a report on the activities undertaken and sent it to the professors and at the end of the educational course, post-test was given. The duration between pretest and post-test was seven days. The questionnaire included knowledge and attitude toward aging and knowledge, and attitude and practice related to oral health of the elderly people. Data were analyzed in SPSS (version 21) software using covariance, paired t-test and regression tests.

\section{Result}

Pre-test and post-test questionnaires were completed by 64 students, among which 28 were boys and 36 were girls. Average ages of students participating in the traditional and virtual education groups were $23.9 \pm 0.72$ and $24.1 \pm 1.2$ years, respectively. The lowest age of participants in both groups was 23; while the highest ages were 29 years in the virtual training group and 25 years in the traditional education group. Pre-intervention scores for both groups in the general knowledge section of old age was $41.7 \pm 45.6$ and $41.7 \pm 19$ (out of 100 ), respectively; whereas in the field of knowledge related to oral health of the elderly, it was $54.2 \pm 19$ and $49.2 \pm 18.2$, respectively. In addition, in the general attitude toward old age, traditional and virtual education groups scored $67.7 \pm 6.8$ and $68.1 \pm 9.3$, respectively.

Moreover, pre-intervention scores in the section of oral health of the elderly in traditional and virtual education groups were $76.8 \pm 12$ and $78.5 \pm 13.2$, respectively. In the self-expressive performance section these scores were $45.4 \pm 20.7$ and $52.9 \pm 20.3$, respectively. There was no significant difference between the two groups in terms of the mean and standard deviation of pre-test scores.

Regression test was used to examine the relationships between underlying factors and knowledge scores and also between attitude and performance of students before the intervention. Background variables included age, gender, marital status, settlement status, living with an elderly person, the history of treating elderly patients, first-degree relatives being dentist, being interested in the field of study, and the average. None of the underlying variables had significant relationship with the general knowledge of old age, knowledge of oral health of the elderly people, attitude toward elderly oral health, and oral health function in old age.

Only the general attitude associated with old age found to have relationships with gender factor $(\mathrm{P}=0.009)$ and "life with an elderly person at the moment" $(\mathrm{P}=0.044)$. Girl students and those who lived with an elderly person at that moment had a better attitude. Covariance test was used to investigate the effect of traditional and virtual teaching methods on post-test scores of students in terms of the level of knowledge, attitude, and performance of students. Difference in scores after intervention by traditional and virtual learning group was not significant in any of the areas examined except for selfexpressive performance related to oral health function in old age. There was a significant difference between 
the scores in the self-explanatory section between the virtual training and the traditional education groups after intervention $(\mathrm{P}=0.017)$.

\section{Conclusion}

The developments in medical knowledge along with a variety of teaching and learning approaches have created new responsibilities for managers and instructors in the higher education system. One of the main pillars of this responsibility is the choice of the most appropriate method of teaching and learning. The results of this study show that virtual training can meet the educational goals in dental topics including "senior dentistry" section. Our results suggest that given the benefits of virtual training, it can be used as a substitute for the traditional teaching style in teaching elderly dentistry topics. In this study, there was no improvement in attitude levels of students in both groups compared with their level of attitudes before intervention. It appears that change in the attitude of students requires longer training programs compared with the elderly attitudes. Increasing educational-therapeutic communication and adding practical training fields for students in society are other approaches for promoting attitude and this will be an introduction in improving performance in this area.

\section{Acknowledgments}

This research was extracted from a MD dissertation in the Department of Community Oral Health, School of Dentistry, Tehran University of Medical Sciences.

\section{Conflict of Interest}

The authors declared no conflicts of interest. 


\title{
طراحى و اجراو ارزيابى آموزش مبحث دندانيزشكى سالمندان به صورت مجازى به دانشجويان

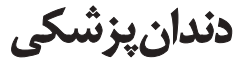

\author{
كتايون سركران'ْه "سمانه رازقى'، زهره خورشيدى'
}

ا. كروه سلامت دهان و دنداثيزشكى اجتماعى، دانشكده دندانيزشكى، دانشكاه علوم يزشكى وخدمات بههاشتىدرمانى تهران، تهران، ايران.

\section{حكدי}

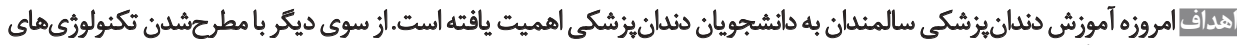

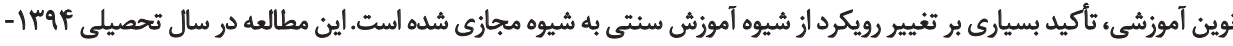

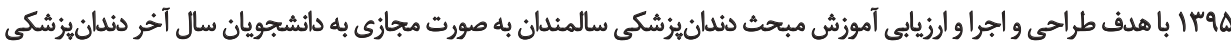

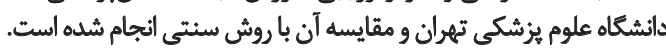

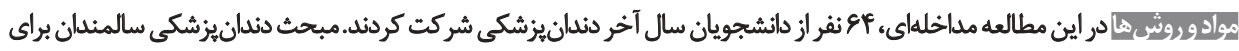

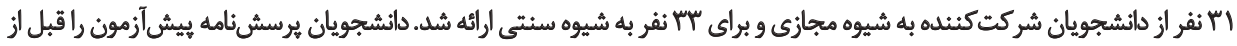

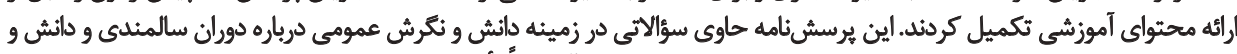

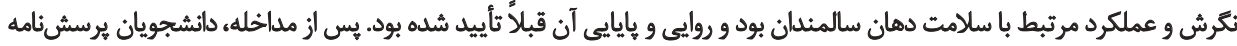

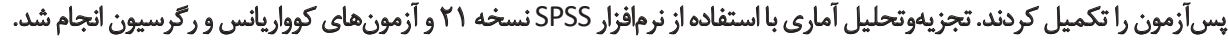

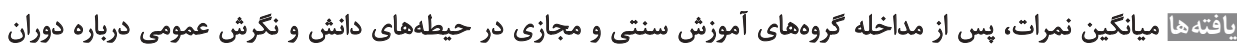

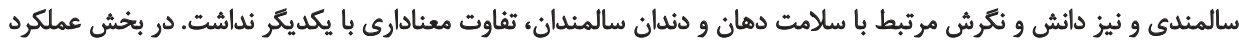

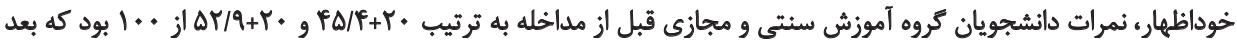

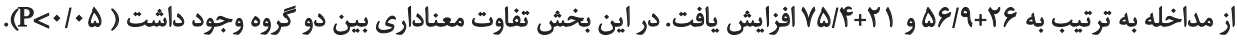

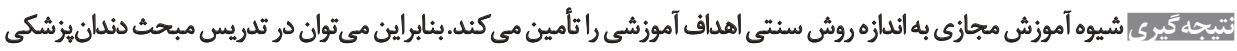

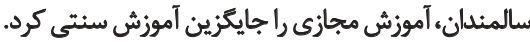

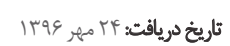

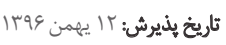

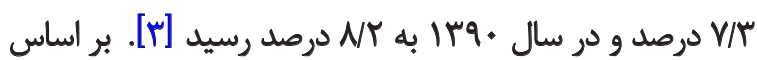

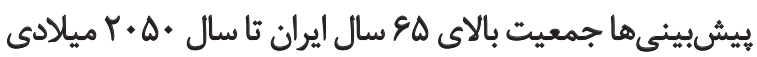

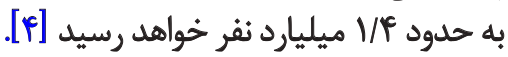

با توجه به روند افزايشى جمعيث سالمندان، لازم است

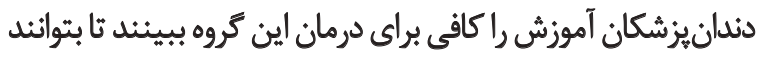

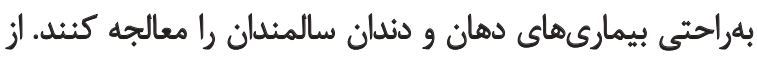

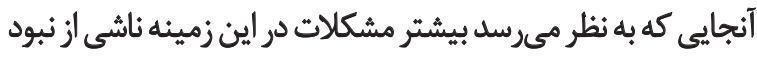

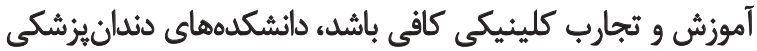

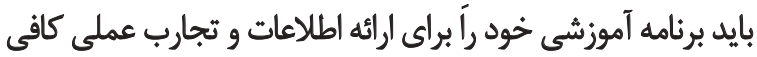

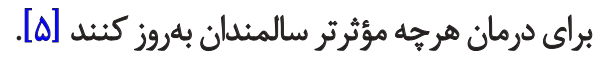

از سوى ديكر، امروزه با كسترش فناورى اطلاعات، ابزارها و ورئ

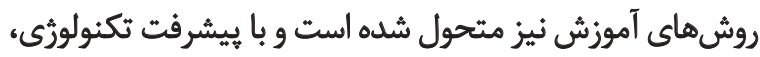

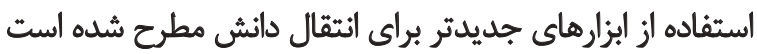

مقدمه

از آنجايى كه برنامهريزىهاي كلان اجتماعى و اقتصادى براي

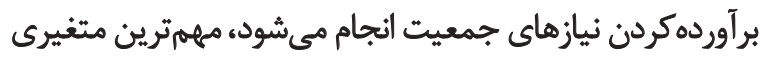

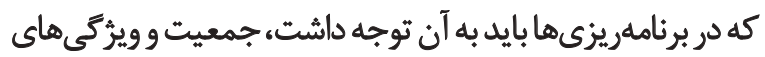

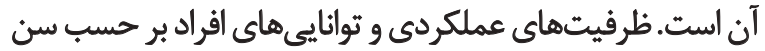

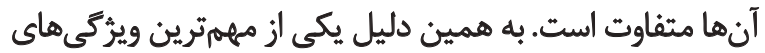

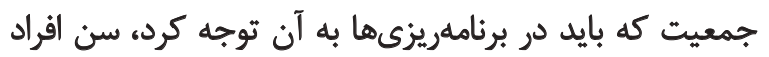

جامعd است [1]

مطالعات همدكيرشناسى نشان ميدهد هماكنون

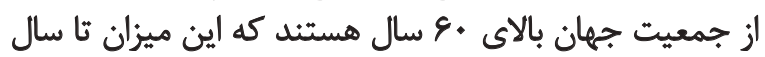

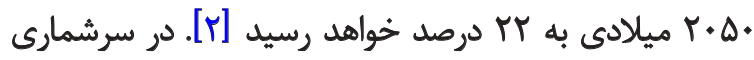

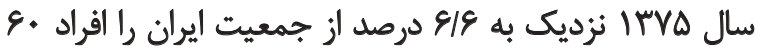

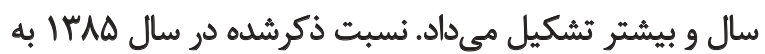


سالمندان و سالمندشناسى بهنازگى به عنوان واندان واحد نظرى به

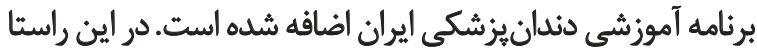

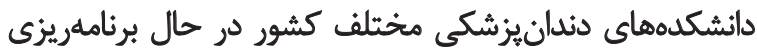
آموزشى هستند. با توجه به اينكه تاكنون مطالعه مانه جامعي انجام

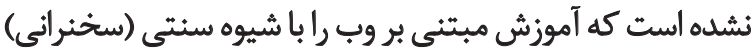

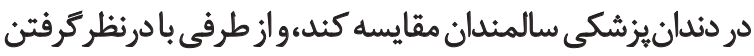

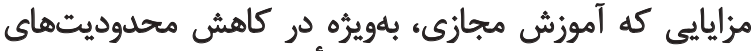

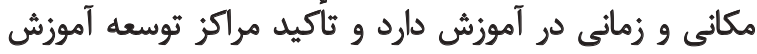

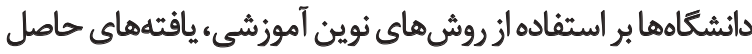

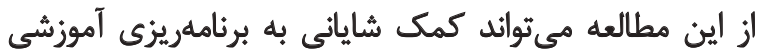

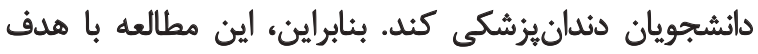

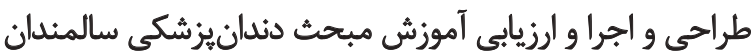

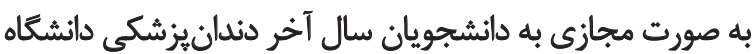

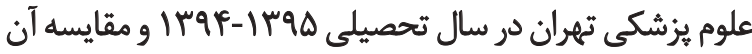

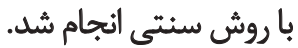

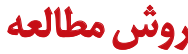

در اين مطالعه تمام دانشجويان ترم آخر رشته دندانيزشكى تركي

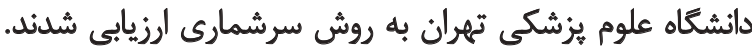

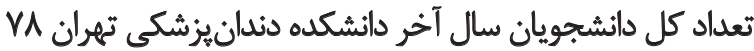

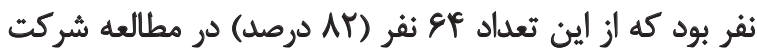

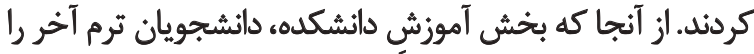

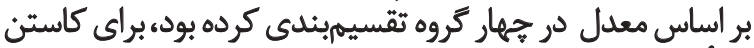

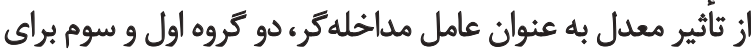

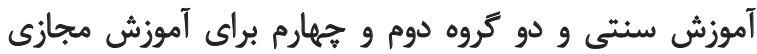

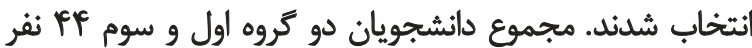

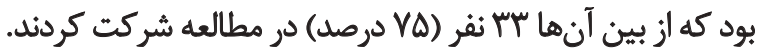

همجنين دانشجويان گروههاى دوم و جهارم، يعنى تروه

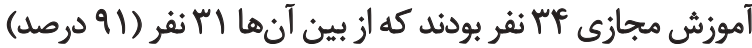

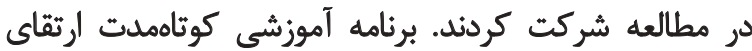

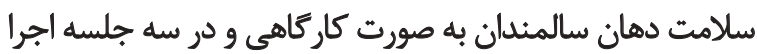

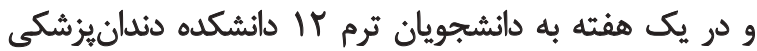

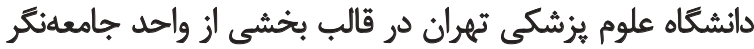

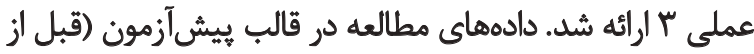

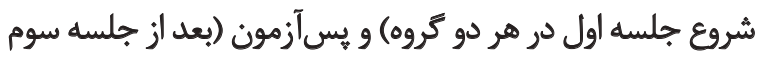

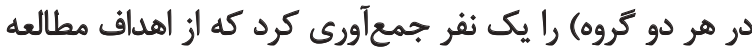

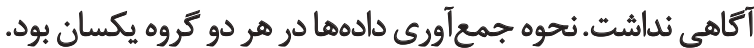

قبل از شروع جلسه اول، يرسش قامنامه بيش آزمون براى تكميل در

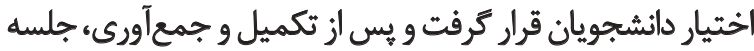

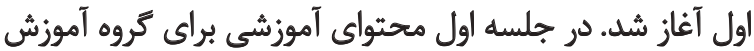

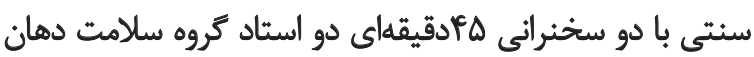

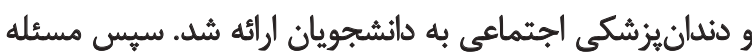

[9]. در دهلهاى اخير تلاشهاى زيادى در كشور شده است تا

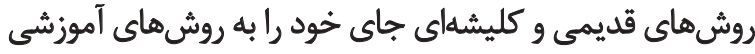

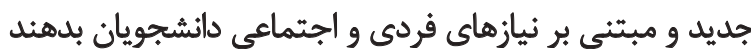

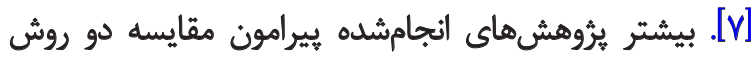

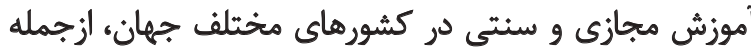

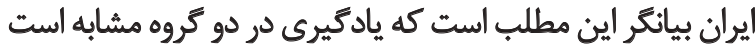

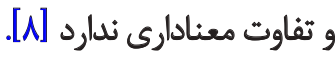

مواردى هميجون كاهش محدوديتهاى مكانى و زمانى براى

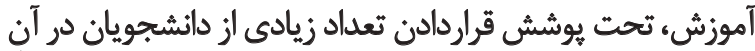

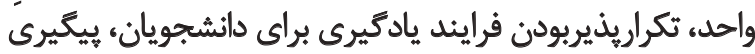

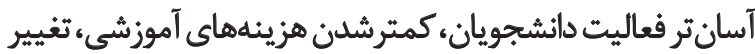

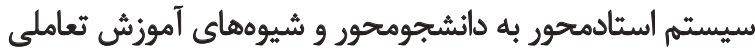

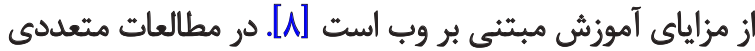

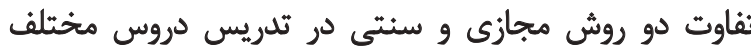

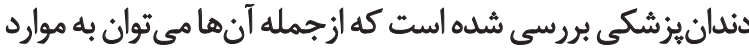

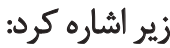

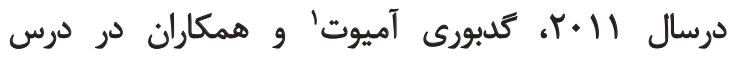

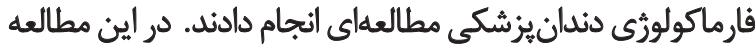

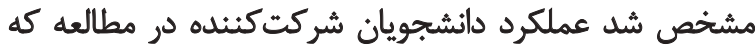

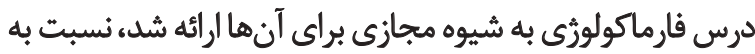

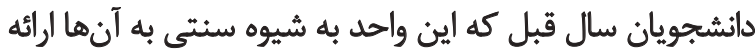

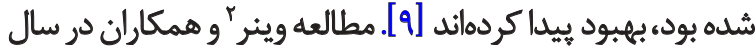

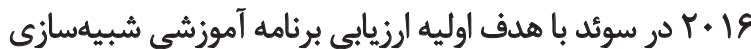

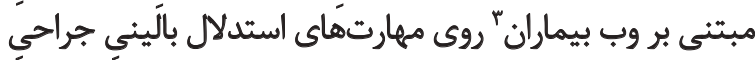

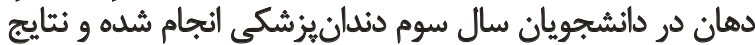

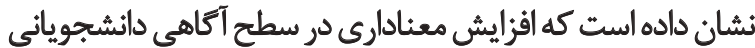
كه مطابق با اهداف آموزشى بود، رخ داده است [ـ [1].

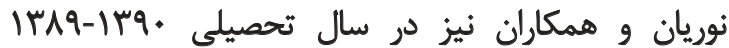

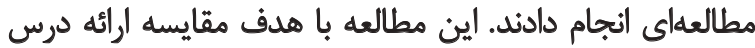

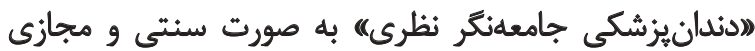

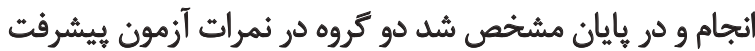

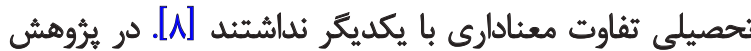

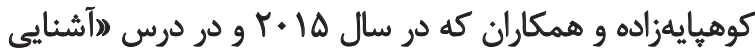

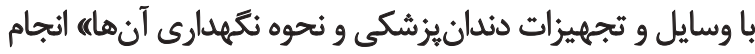

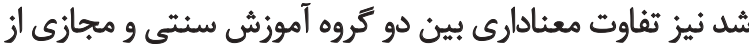
نظر نمرات أكاهى سنجي و عملكرد وجود نداشت بين [11]. بيشتر مطالعاتى كه بيرامون بررسى تأثير روش مجازى در دراني

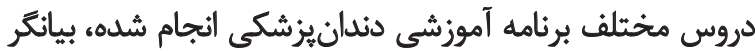

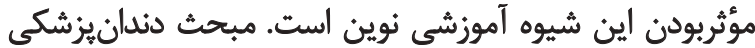

1. Gadbury-Amyot

2. Weiner

3. Web-Sp 


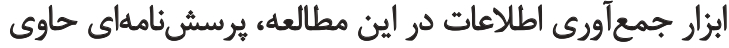

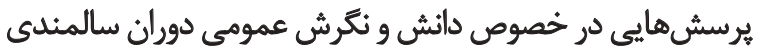

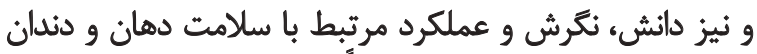

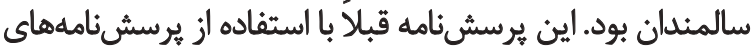

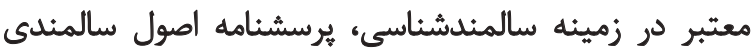

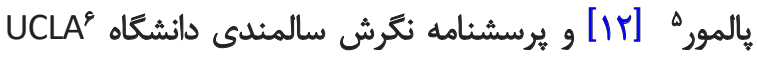

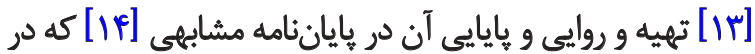

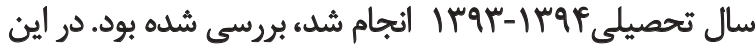

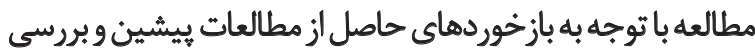

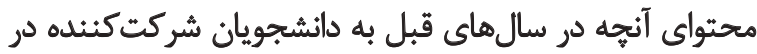

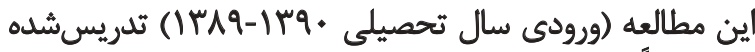

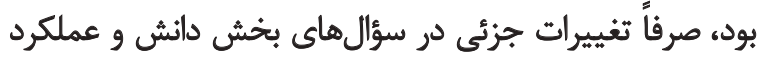

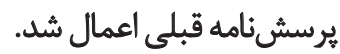

بخشهاى اين يرسشنامه عبارت بود از: اطلاعات زمينهاي

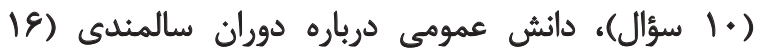

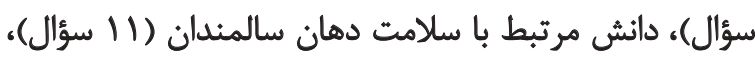

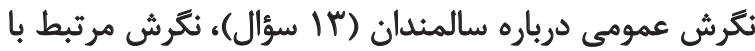

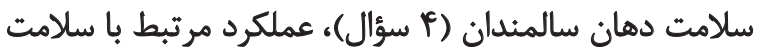

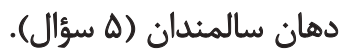

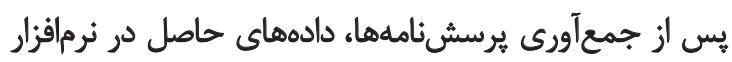

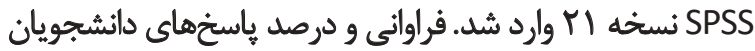

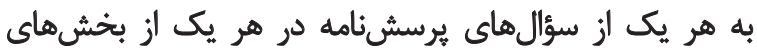

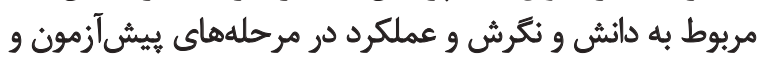

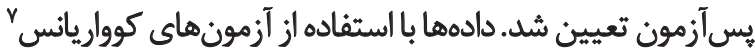
و تى زوجى ^و ركرسيون " تجزيهوتحليل شد.

\section{يافتهها}

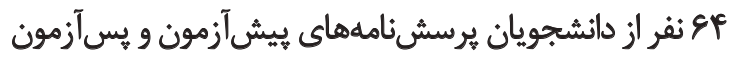

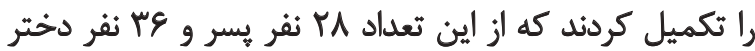

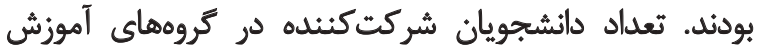

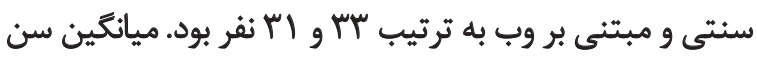

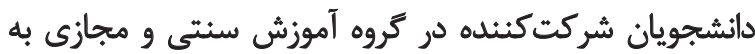

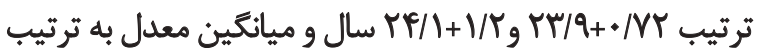

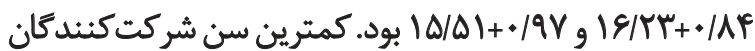

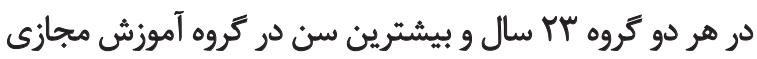

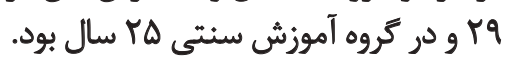

نمرات بيش از مداخله دو كروه آموزش سنتى و مجازى در دراني

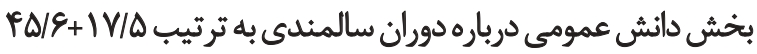

5. Palmore's Facts On Aging

6. UCLA Geriatric Attitude Scale (GAS)

7. Co-Variance

8. Paired t-test

9. Regression
(بيمارنماى كاغذى)" طراحى شده بيان و از دانشجويان خواسته

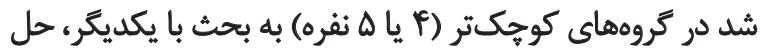

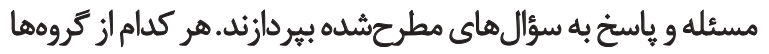

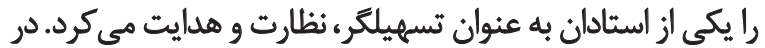
انتها ياسخ سؤال ها در اختيار دانشجويان قرار ترفت.

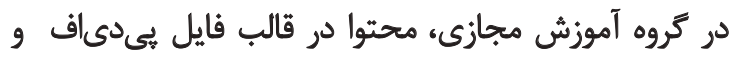

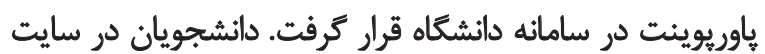

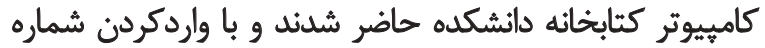

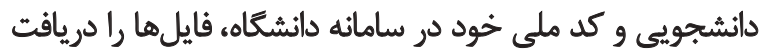

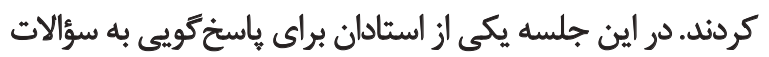

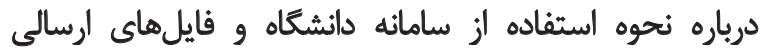

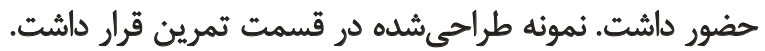

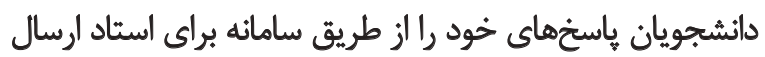

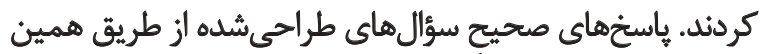

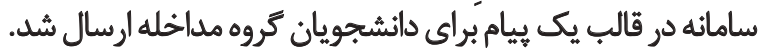

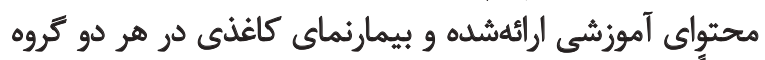

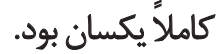

محتواى آموزشى ارائهشده به دانشجويان شامل دو بخش بود:

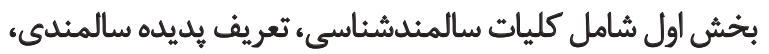

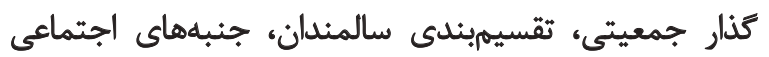

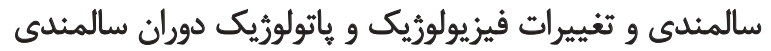

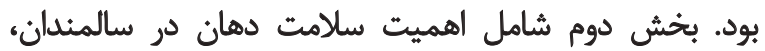

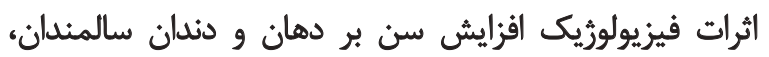

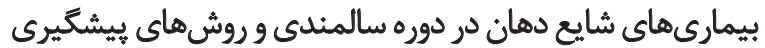
از بيمارىهاي دهان و دندان در سالمندان بوده.

جلسه دوم برنامه آموزشى براي دانشجويان هر دو كروه سنتى

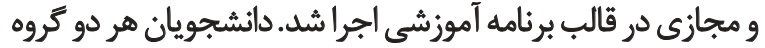

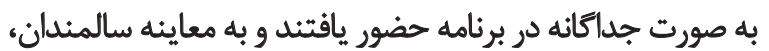

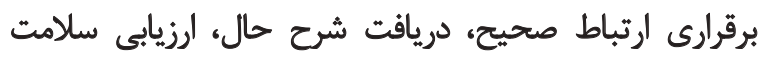

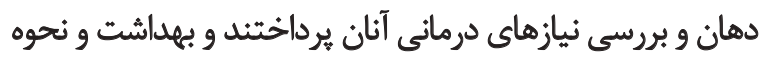

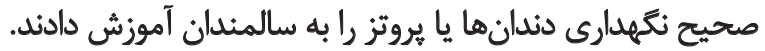

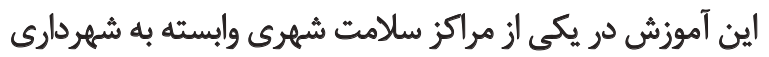

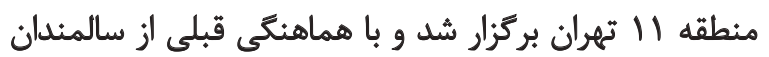

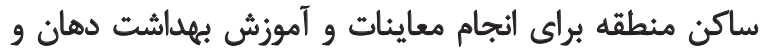

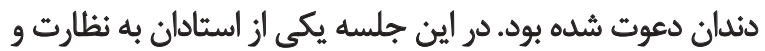

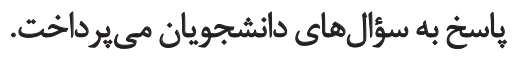

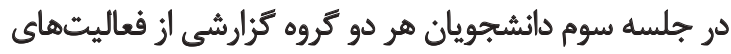

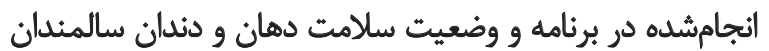

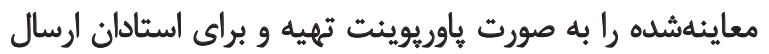

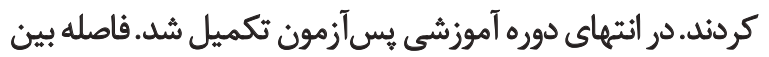

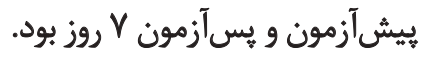

4. Paper case 


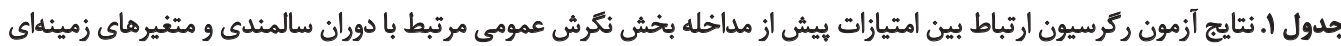

\begin{tabular}{|c|c|c|c|}
\hline $\mathbf{P}$ & بثا & B & متغير \\
\hline.$M T P$ & $-+1+\Delta A$ &.$- / 48$ & سن \\
\hline $.1 . .9^{*}$ &.$- / P \pi T$ & $-\varphi / P$ & جنسيت \\
\hline .1814 & $-+1 \cdot n^{n}$ & $-* / M^{p}$ & وضعيث ثأهل \\
\hline . Mrq & $.1 \cdot \mathrm{FV}$ & $\cdot / \pi f$ & محل سكونت \\
\hline 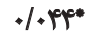 &.$/ 499$ & $-r / \cdot \gamma$ & زندكى با فرد سالمند در حال حاضر \\
\hline.$/ 901$ & 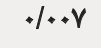 & $+/ 10$ & سابقه معاينه يا درمان بيمار سالمند \\
\hline . 1894 & -.1 .84 & $-\cdot /$ Af & دنلانيزئشكبودن بستكان درجه يكى \\
\hline$\cdot / T \Delta \Delta$ & +/IAT & $r / r)$ & عالاقهندى به رشته \\
\hline MfFe & $-\cdot / r \Delta A$ & $-1 / M r$ & معدل معل \\
\hline
\end{tabular}

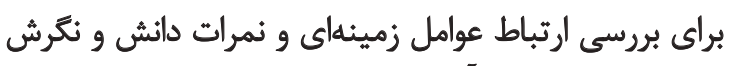

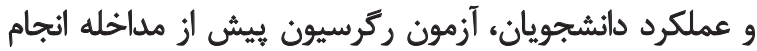

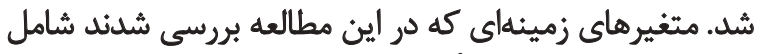

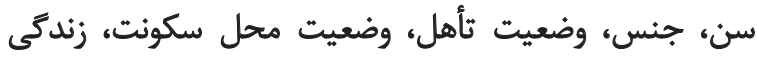
با فرد سالمند در حال حاضر، سابقه وضعيت درمان بيماران سان سالمند،

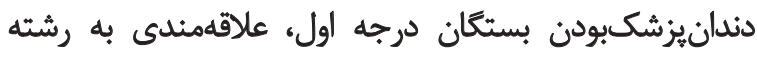

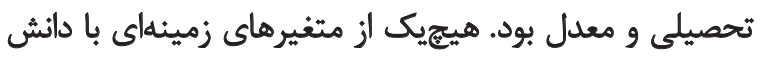

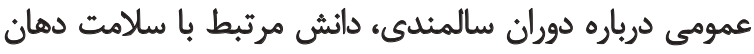

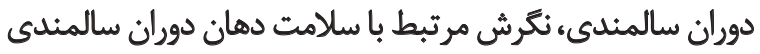

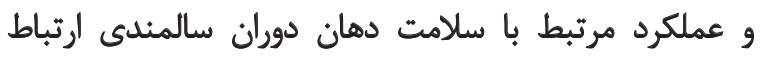

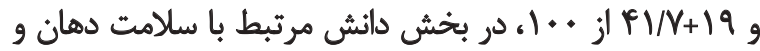

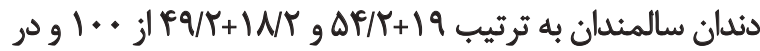

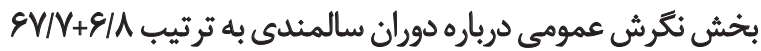

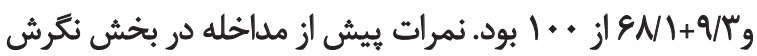

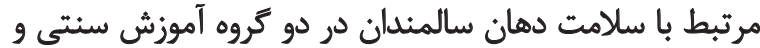

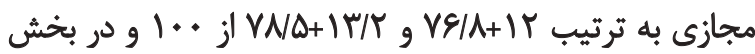

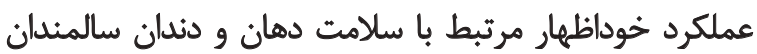

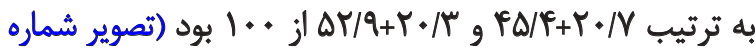

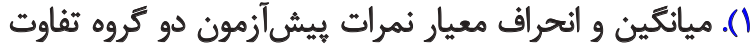

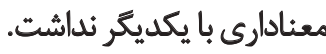

جدول r. تثتايج آزمون تى زوجى براي مقايسه تغييرات متغيرها قبل و بعد از مداخله

\begin{tabular}{|c|c|c|c|c|c|}
\hline $\mathbf{P}$ & $\mathbf{t}$ & انحراق معيار & مياتئين & روش آموزشى & \\
\hline$\%$ & $-q \Delta E$ & $r|q|$ & $-r / r q$ & دانش عمومى قبل و بعد & \\
\hline$\%$ & $-F / f$ & $r / T \Delta$ & $-1 / N$ & دانش مرتبط با سلامت دهان ثبل و بعد & \\
\hline.$/ 14$ & I/ra & $F / F q$ & $1 / \bullet$ & نكرش عمومى قبل و بعد & سنتى \\
\hline.$/ 4 q$ & $y / \cdot v$ & $t / F r$ &.$/ F \Delta$ & نكرش مرتبط با سلامت دهان قبل و بعد & \\
\hline $.1 \cdot r$ & $-T / P Q$ & $1 / m$ & $-\infty / \Delta V$ & عملكرد & \\
\hline$\%$ & $-\$ / 9 r$ & $r / \Delta 1$ & $-f / A$ & دانش عمومى قبل و بعد & \\
\hline$\%$ & - p/pa & $m / .8$ & $-r / R \theta$ & دانش هرتبط با سلامت دهان قبل و بعد & \\
\hline .198 &.$/ 4 T^{\circ}$ & P/M & $\cdot / M T$ & نكرش عمومى قبل و بعد & مجازي \\
\hline .11 & V/gV & $r / r \omega$ &.$/ v$ & نكرش مرتبط با سلامت دهان قبل و بعد & \\
\hline$\%$ & $-\Delta / 1$ & $1 / \pi r$ & $-1 / M r$ & عملكرد & \\
\hline
\end{tabular}




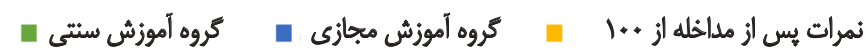

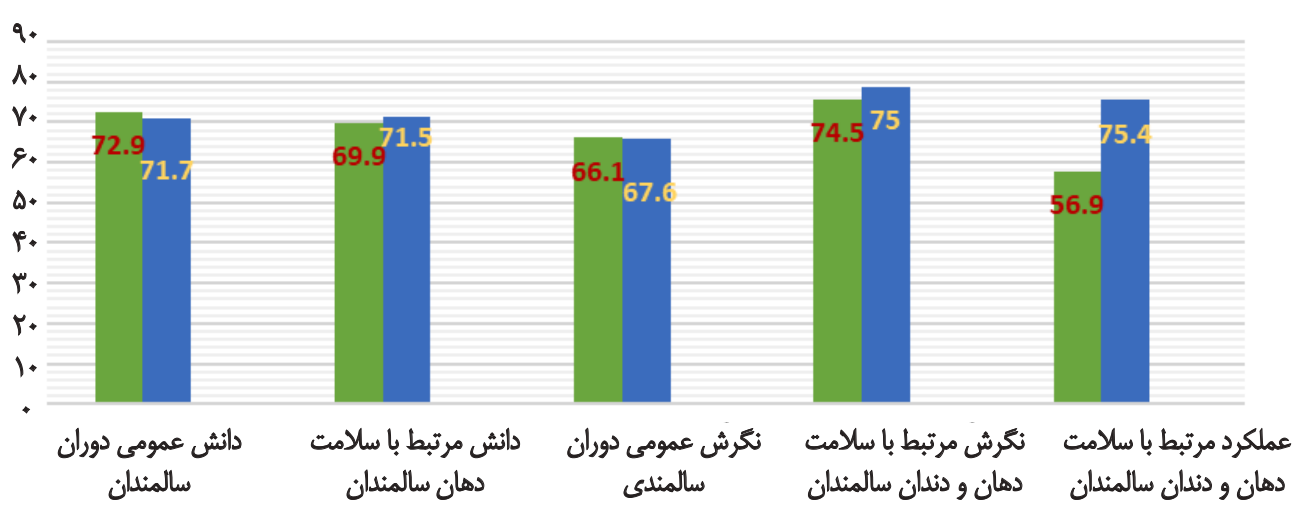

䀡

تصوير ا. نمودار مقايسهاي نمرات يس از مداخله دانشجويان كروه آموزش مجازى و سنتي سني

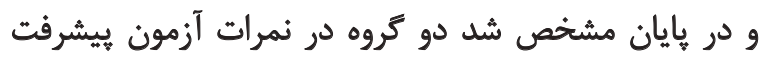

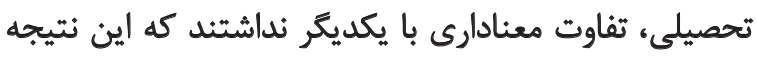

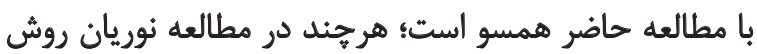
ارزيابى با مطالعه حاضر متثاوت بود.

يافتههاى يُوهش كوهيايهزاده و همكاران [11].كه در سال

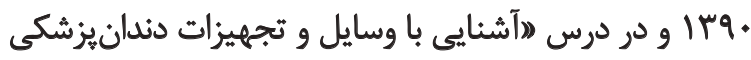

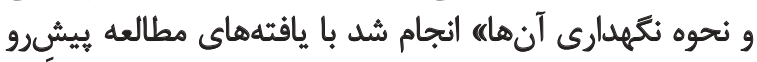

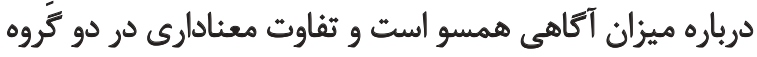

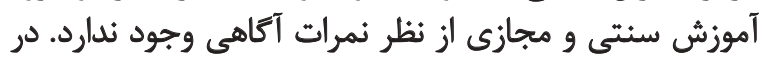

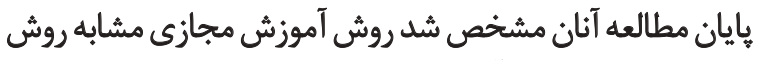

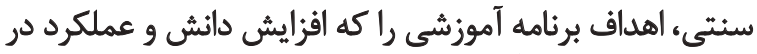

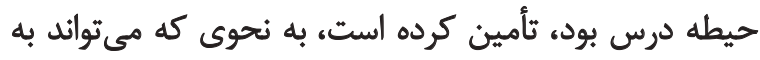

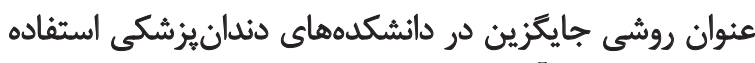

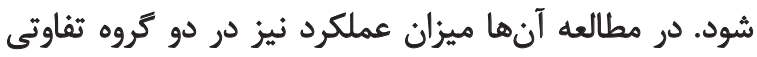

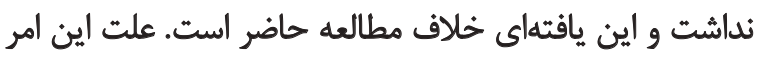
مى توائد تفاوت در نحوه سنجش عملكرد در دو دو مطالعه باشدي؛

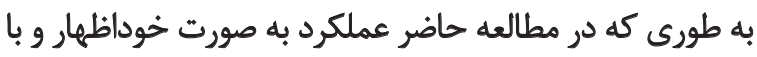

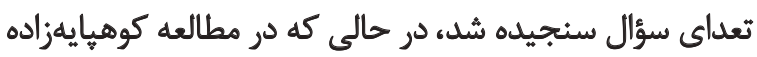

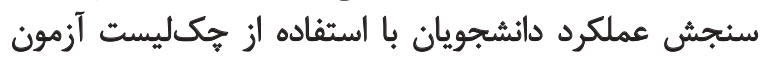

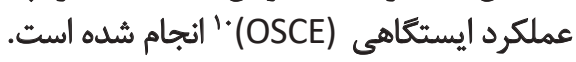

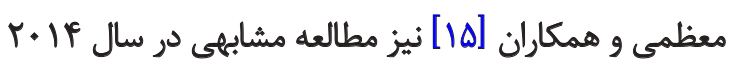

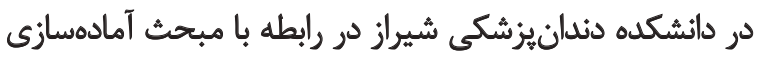

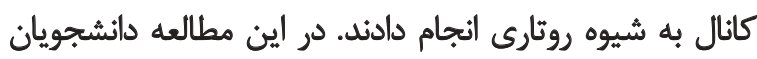

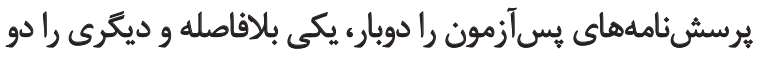

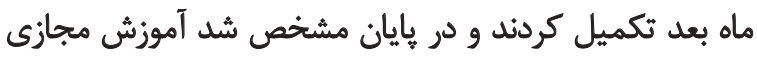

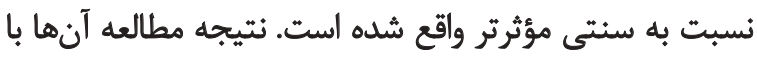

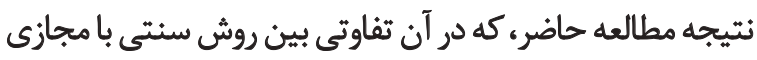

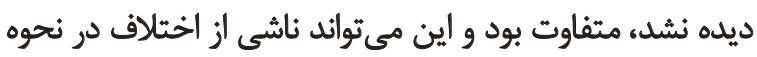

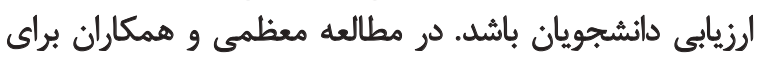

10. Objective Structured Clinical Examination معنادارى نداشتند. فقط نغرش عمومى مرتبط با دوران سالمندى

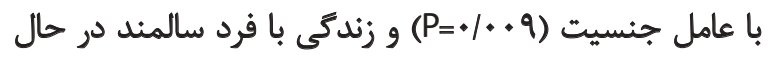

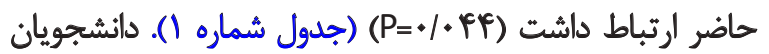
دختر و دانشجويانى كه در حال حاضر با فر فرد سالمند زندكى

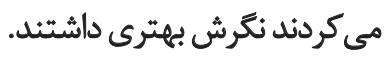
براى بررسى ثأثير دو روش آموزش سنتى و مجازى بر سطح

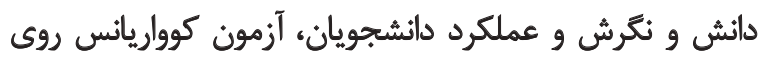

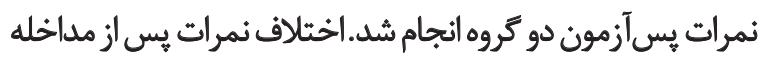

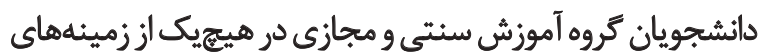

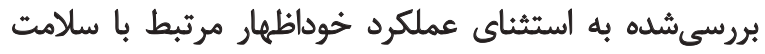

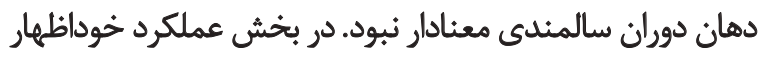

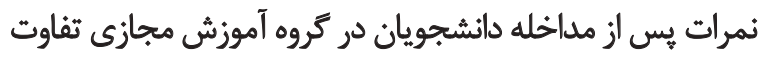

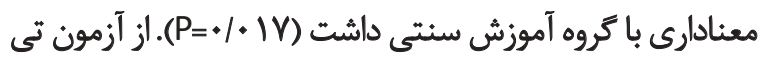

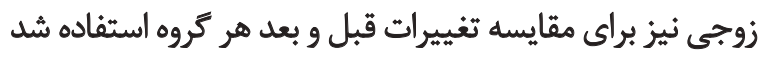

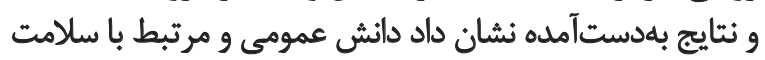

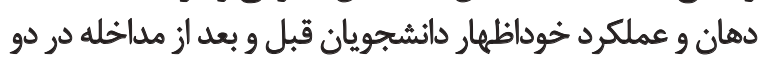

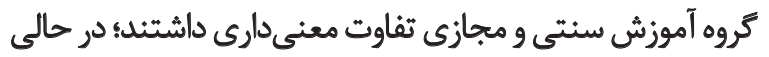
كه نكرش عمومى و مرتبط با سلامت دهان قبل و و بعد از مداخله

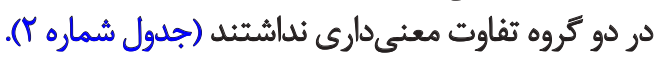

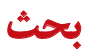

نتايج مطالعه حاضر نشان داد آكاهي و نكرش دانشجويان دو كروه در زمينه دوران سالمندى و سلامت دهان دان دان سالمندان

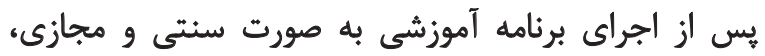

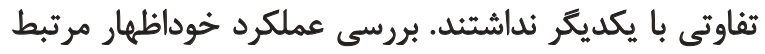

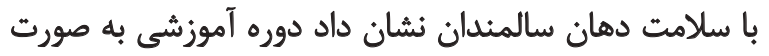

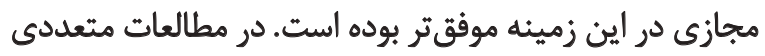

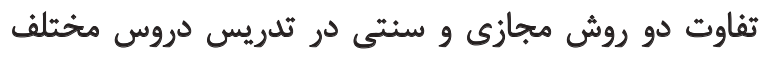

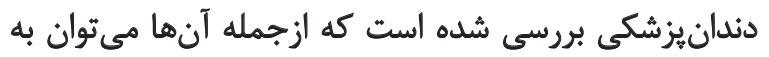

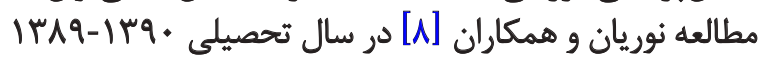

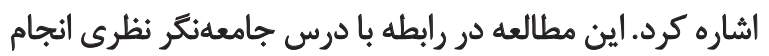


كُّشته، سابقه درمان بيماران سالمند، دندانيز شككبودن بستكان

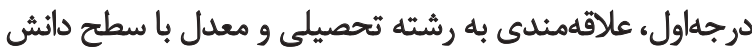

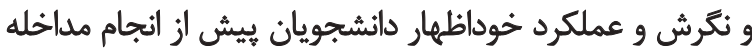

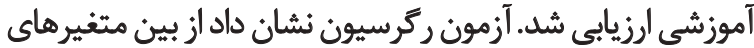

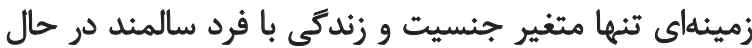
حاضر با متغير نكرش عمومى دوران سالمندى جنه ارتباط داشتند. درباره ارتباط عوامل زمينهاي با دانش و نتُرش دانشجويان

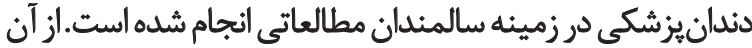

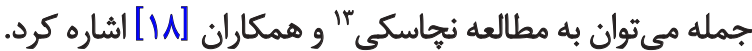

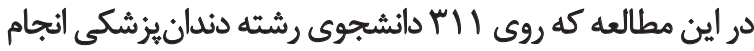

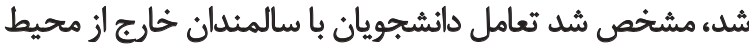

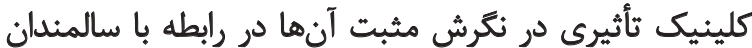

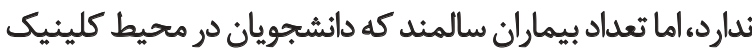

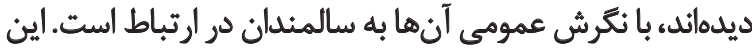
يافتهها با مطالعه بيشرو درو يكى راستا نيستند.

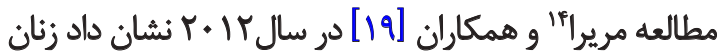

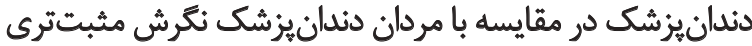

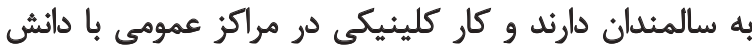

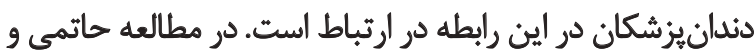

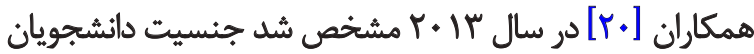

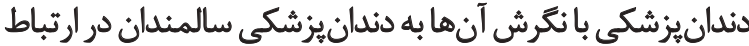

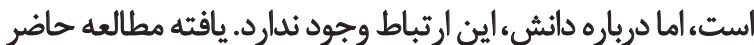

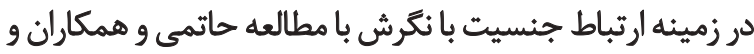

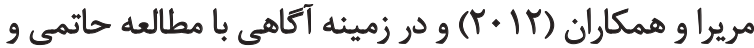

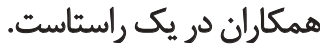

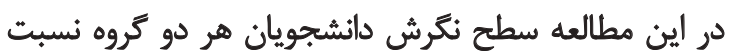

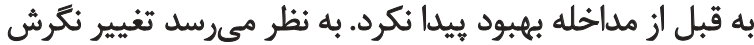

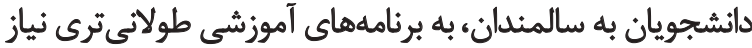

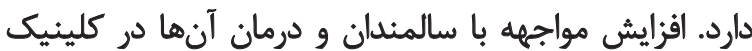

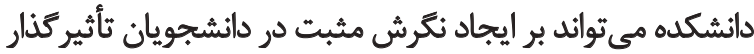

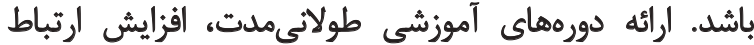

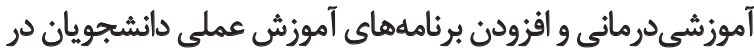

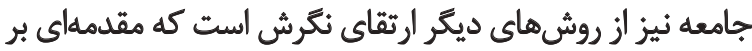

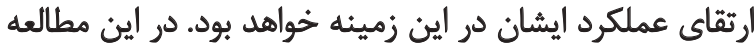

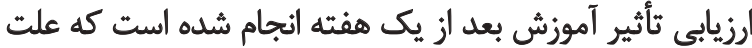

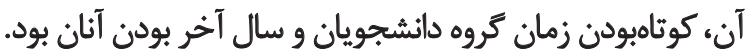

\section{نتيجلَيرى نهايى}

رشد وييوسته دانش يزشكى همراه با رويكردهاي متعدد آموزش

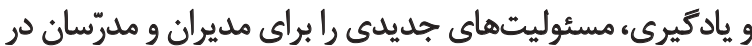

13. Nochaski

14. Moreira
ارزيابي، صرفاً ز يسآزمون استفاده شده بود.

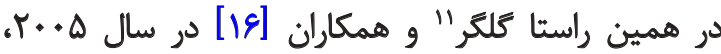

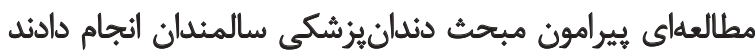

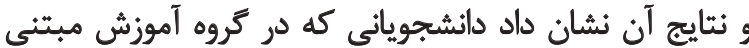

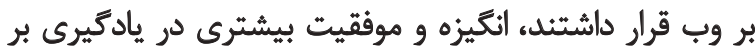

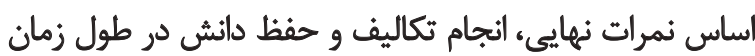

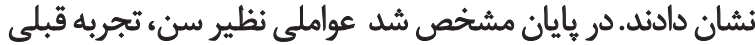

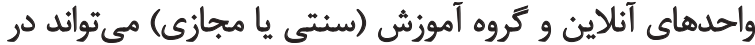

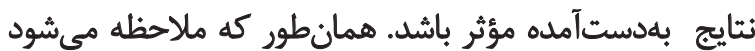

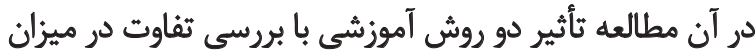

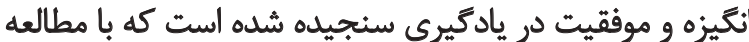

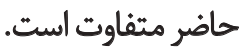

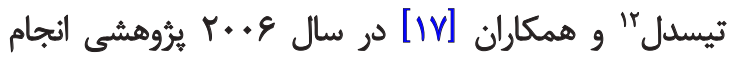

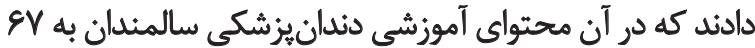

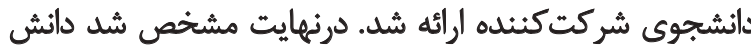

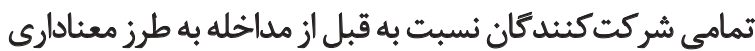

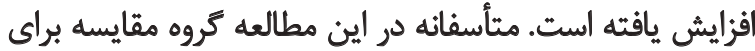

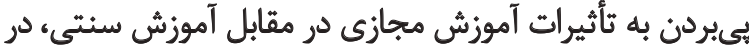

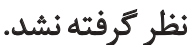

يكى ديكر از مطالعاتى كه در زمينه مقايسه آموزش مجازى إنى

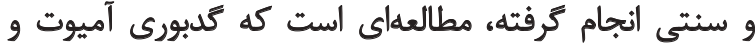

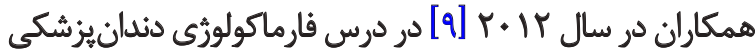

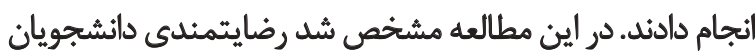

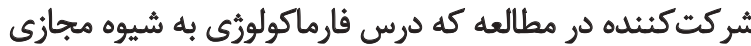

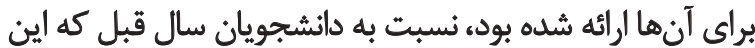

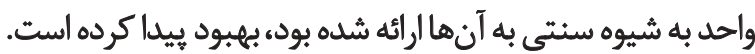

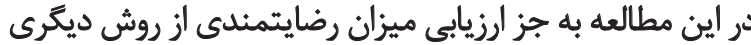

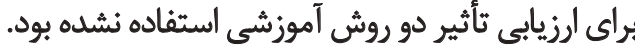

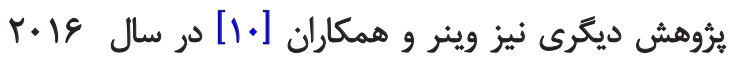

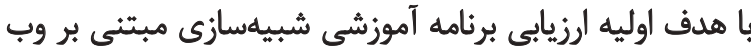

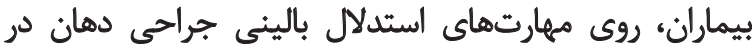

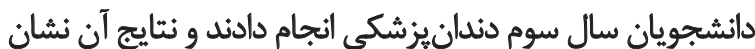

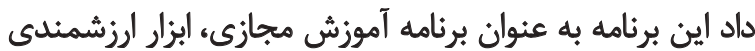

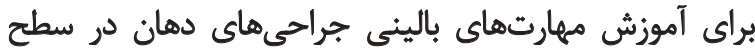

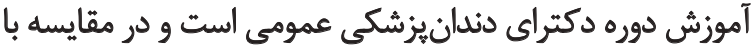

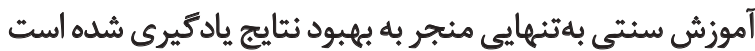
كه با مطالعه حاضر همسو است.

در مطالعه بيشروو وجود ارتباط بين يكسرى ازعوامل زمينهاى

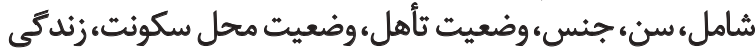

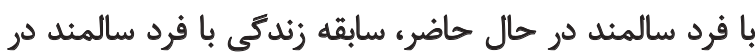


نظام آموزش عالى ايجاد كرده است. يكى ازٔ محورهاي اصلى اين

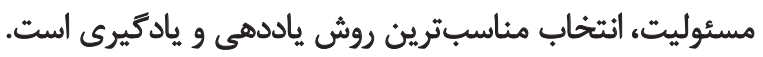

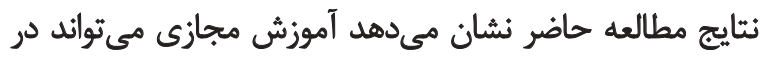

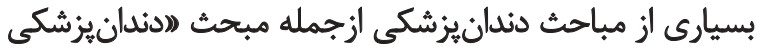

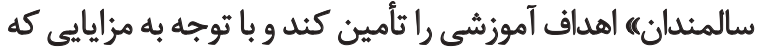

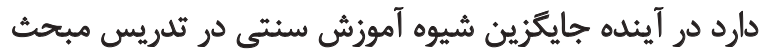

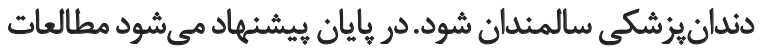

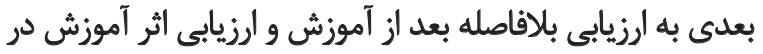

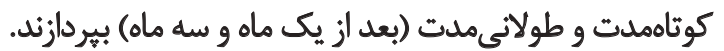

$$
\text { تشكر و قدردانى }
$$

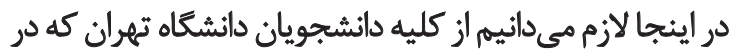

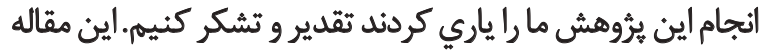

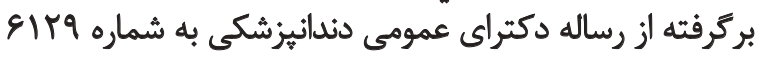

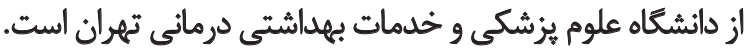




\section{References}

[1] Azizi Zeinalhajlu A, Amini A, Sadeghtabrizi J. [Consequences of population aging in Iran with emphasis on its increasing challenges on the health system (Literature review) (Persian)]. Depiction of Health. 2015, 6(1):54-64.

[2] Kanasi E, Ayilavarapu S, Jones J. The aging population: demographics and the biology of aging. Periodontology 2000. 2016; 72(1):13-8. doi: 10.1111/prd.12126

[3] Statis tics Center of Iran. [Results of national census according age groups (Persian)]. Tehran: Statistics Center of Iran; 2018.

[4] United Nations. World population prospects 2017: Population aged 65 and over. New York: United Nations; 2017.

[5] Levy N, Goldblatt RS, and Reisine S. Geriatrics education in US dental schools: Where do we stand, and what improvements should be made? Journal of Dental Education. 2013; 77(10):127085. PMID: 24098031

[6] Fathi Vajargah K, Jahani SH, Azadmanesh N. Application of icts in teaching and learning at university level: The case of Shahid Beheshti University. Turkish Online Journal of Educational Technology. Turkish Online Journal of Educational Technology. 2010; 9(2):33-39.

[7] Shahsavari Isfahani S, Mosallanejad L, Sobhanian S. The effect of virtual and traditional methods on students learning and competency - based skills. Hormozgan Medical Journal, 2010; 14(3):184190

[8] Nourian A, Nourian A, Ebnahmadi A, AkbarzadehBaghban A, Khoshnevisan $\mathrm{MH}$. [Comparison of e-learning and traditional classroom instruction of dental public health for dental students of Shahid Beheshti Dental School during 2010-2011 (Persian)]. Journal of Dental School. 2012; 30(3):174-183.

[9] Gadbury-Amyot CC, Brockman WG. Transition of a traditional pharmacology course for dental students to an online delivery format: A pilot project. Journal of Dental Education. 2011; 75(5):633-45. PMID: 21546597

[10] Weiner CK, Skålén M, Harju-Jeanty D, Heymann R, Rosén A, Fors $U$, et al. Implementation of a web-based patient simulation program to teach dental students in oral surgery. Journal of Dental Education. 2016; 80(2):133-40. PMID: 26834130

[11] Kouhpaiezadeh J, Khoshnevisan MH, Beiranvand A. [Comparison of the two Virtual and Traditional teaching methods inlearning the course of the "Introduction to Dental equipment and theirmaintenance" for the students of the PhD General dentistry at Shahid Beheshti University of Medical Sciences (Persian)]. Razi Journal of Medical Sciences, 2015; 23(143):63-70

[12] Fabiano JA, Waldrop DP, Nochaski TH, Davis EL, Goldberg LJ. Understanding dental students' knowledge and perceptions of older people: Toward a new model of geriatric dental education. Journal of Dental Education. 2005; 69(4):419-33. PMID: 15800255

[13] Stewart TJ, Roberts E, Eleazer P, Boland R, Wieland D. Reliability and validity issues for two common measures of medical students' attitudes toward older adults. Educational Gerontology. 2006; 32(6):409-21. doi: 10.1080/03601270600685628

[14] Mohebbi SZ. [Implementation and evaluation of theeffectiveness of a short course on geriatric dentistry for senior dental students of Tehran University of Medical Sciences (Persian)] [MD thesis]. Tehran: Tehran University of Medical Sciences; 2013.
[15] Moazami F, Bahrampour E, Azar MR, Jahedi F, Moattari M Comparing two methods of education (virtual versus traditional) on learning of Iranian dental students: a post-test only design study. BMC Medical Education. 2014; 14:45. doi: 10.1186/14726920-14-45

[16] Gallagher JE, Dobrosielski-Vergona KA, Wingard RG, Williams TM. Web-based vs. traditional classroom instruction in gerontology: A pilot study. American Dental Hygienists Association. 2005; 79(3):7. PMID: 16197772

[17] Teasdale TA, Shaikh M. Efficacy of a geriatric oral health CD as a learning tool. Journal of Dental Education. 2006; 70(12):1366-9. PMID: 17170329

[18] Nochaski TH, Davis EL, Waldrop DP, Fabiano JA, Goldberg LJ. Dental Students' attitudes about older adults: Do type and amount of contact make a difference? Journal of Dental Education. 2011; 75(10):1329-32. PMID: 22012776

[19] Moreira AN, Rocha ÊS, Popoff DA, Vilaça ÊL, Castilho LS, De Magalhães CS. Knowledge and attitudes of dentists regarding ageing and the elderly. Gerodontology. 2012; 29(2):e624-31. doi: 10.1111/j.1741-2358.2011.00534.x

[20] Hatami B, Ahmady AE, Khoshnevisan MH, Lando HA. Senior dental student's attitudes toward older adults and knowledge of geriatric dental care in the Islamic Republic of Iran. Eastern Mediterranean Health Journal. 19(3):S172-7. PMID: 24995742 
